\title{
A Novel Approach for the Intranuclear Cascade
} Joshua Isaacson*, William Jay*, Alessandro Lovato ${ }^{\dagger}$, Pedro Machado*, Noemi Rocco* ${ }^{*}$ Fermi National Accelerator Laboratory, ${ }^{\dagger}$ Argonne National Laboratory

A bstract
- Traditional Intranuclear Cascades assume
point like interactions $\rightarrow$ neglects interaction
range
- Propose new algorithm using:
- Nuclear configurations
- Model for wavefunction overlap
- Compare approach to traditional Mean Free
Path Algorithm in:
- $p C$ cross-sections
- Nuclear transparency

\section{Introduction}

- Intranuclear cascades describe transition from a hard interaction to the final state

- Comprises propagation of nucleons from the inside to the outside of the nucleus

- Essential for understanding:

- Electron-nucleus scattering experiments

- Neutrino oscillation experiments

- Dark Matter searches

- Intranuclear cascades need proper modelling of:

- Hadron-nucleon scattering cross-sections

- Fermi statistics via Pauli Blocking

- Nuclear potential

- Here we propose a new algorithm using:

- Pre-determined nucleon positions

- Pauli blocking effects

- Geometric interpretation of the nucleon-nucleon cross-section

\section{Theory: Configurations}

Generate nuclear configurations according to: (1) Nuclear density

(2) Nuclear correlations (optional)

ษ This can be done using Quantum Monte Carlo [1], 寻 or Mean Field approaches
Theory: Geometric Method for

\section{Cross-Sections}

- Interpret cross-sections as a probability of

interacting via wavefunction overlap

- Require: $P(b=0)=1, b$ is the impact

parameter

- Require: $\int_{0}^{2 \pi} \int_{0}^{\infty} b P(b) d \phi d b=\sigma$

- Model wavefunction overlap modeled with: (1) Cylinder:

$$
P_{\text {cyl }}(b)=\Theta\left(\sigma / \pi-b^{2}\right)
$$

(2) Gaussian:

$$
P_{\text {Gau }}=\exp \left(-\frac{\pi b^{2}}{\sigma}\right)
$$

Algorithm

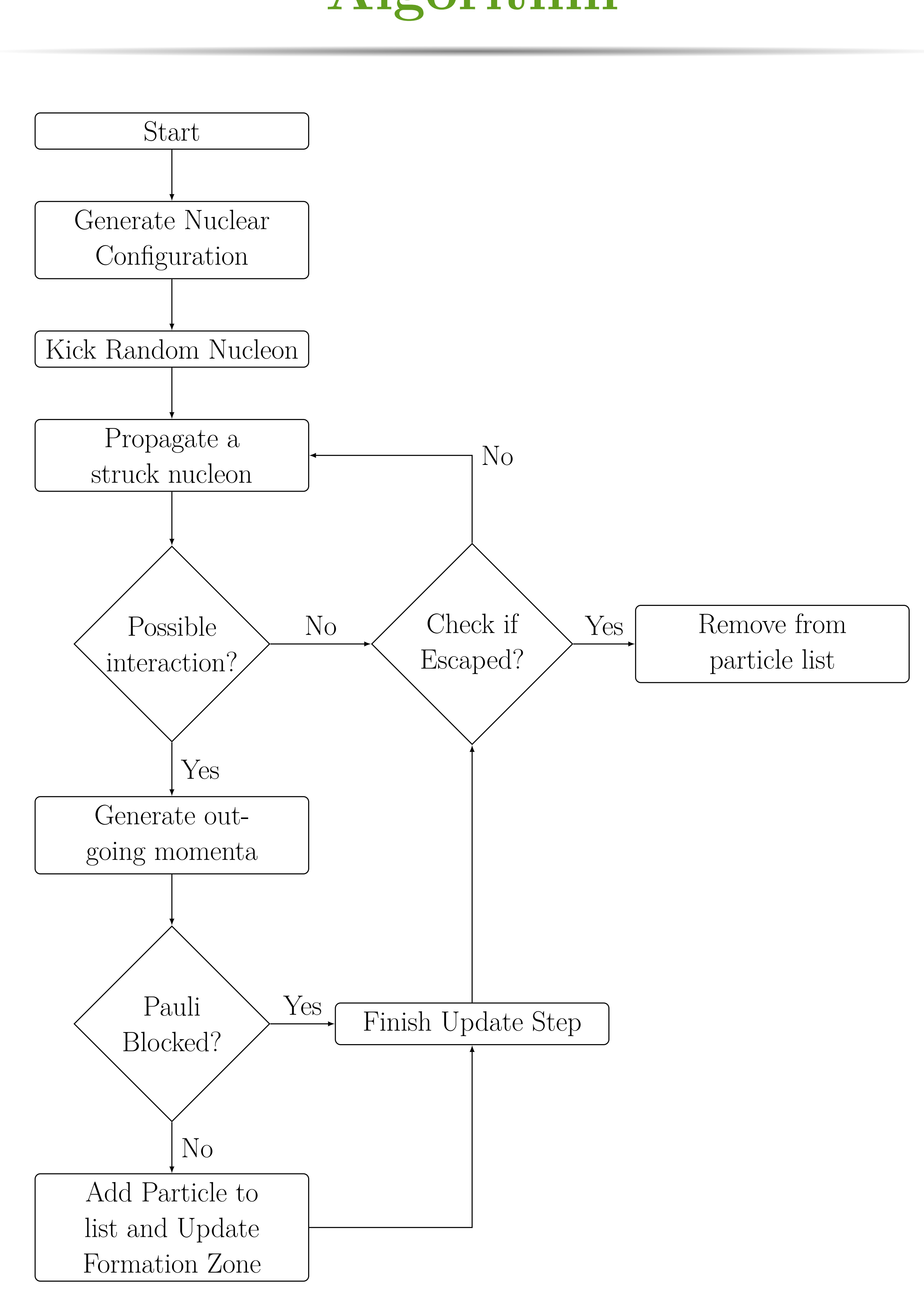

Mean Free Path Validation

Fix density and nucleon cross-sections:

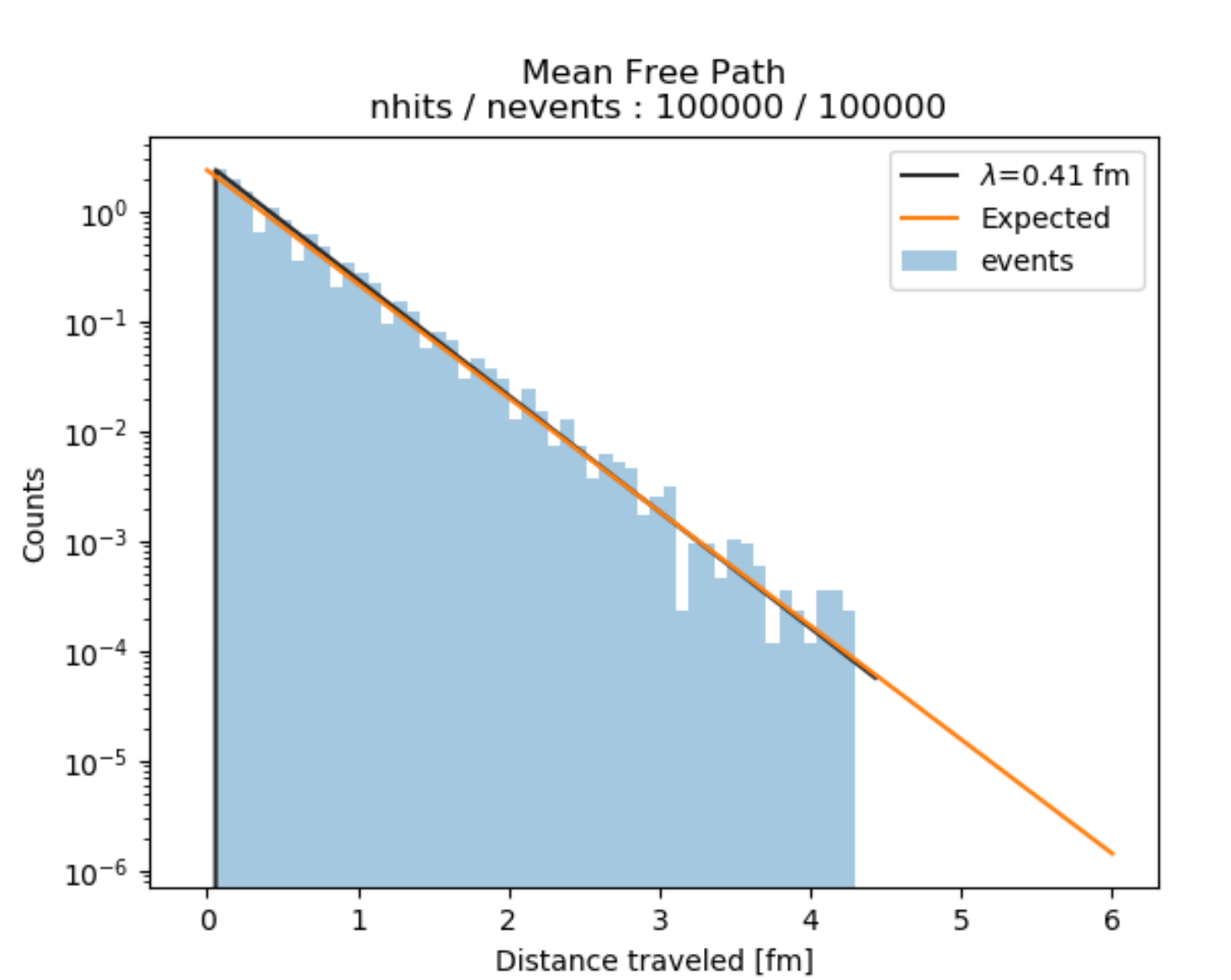

Calculation Set-Up

- Use elastic $p p$ and $p n$ cross-sections [2]

- Calculations considered:

(1) Mean Free Path(MFP) Algorithm [3]

2 Configuration based Algorithm using:

(1) Quantum Monte Carlo (QMC) or Mean Field (MF)

(2) Gaussian or Cylinder interaction probabilities

\section{Cross-Section Results}

- p-Nucleus scattering used to test cascade models

- Two Pieces: $\sigma_{\text {tot }}=\sigma_{r}+\sigma_{e l}$

- Below: Comparison of $\sigma_{r}$ data [4] to different

cascade models for $p+C \rightarrow X$

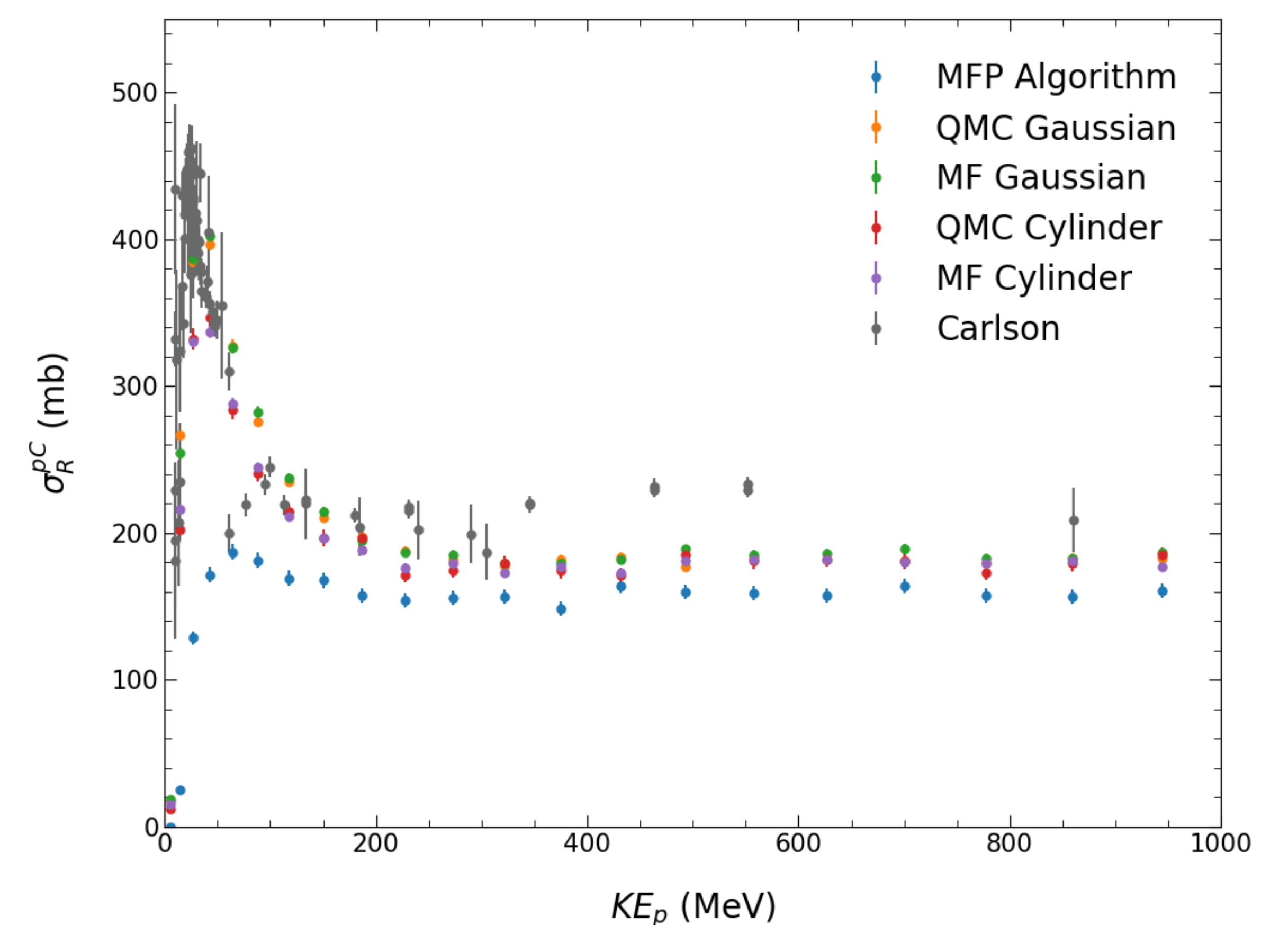

Transparency Results

Transparency $=\frac{N_{n o} F S I}{N_{\text {total }}}$,

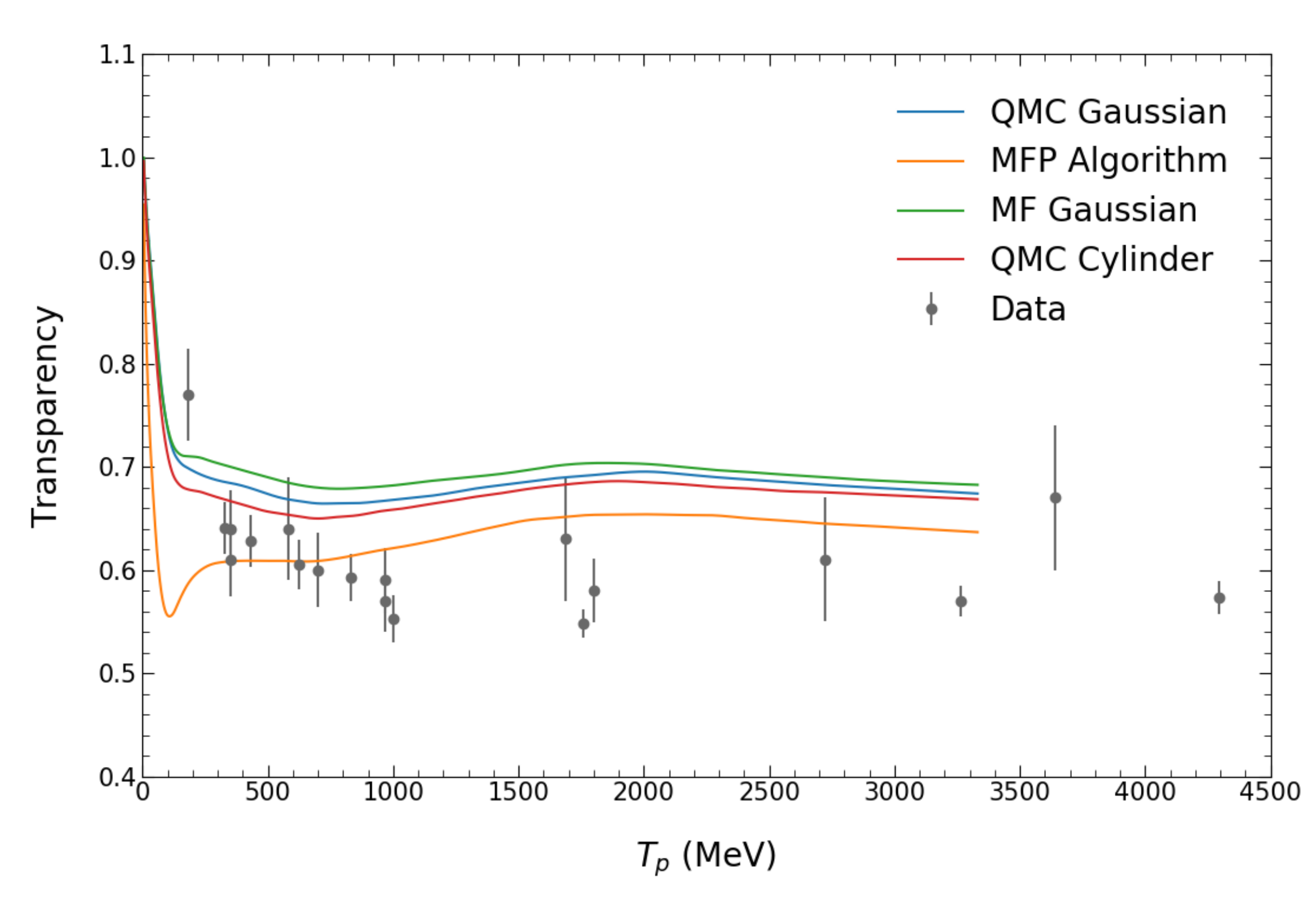

Data is from: $[5,6]$

Conclusion \& Future Work

- Proposed new algorithm based on configurations for cascades

- Include the effects of inelastic scattering (pion cascade), which is important at high energy

\section{References}

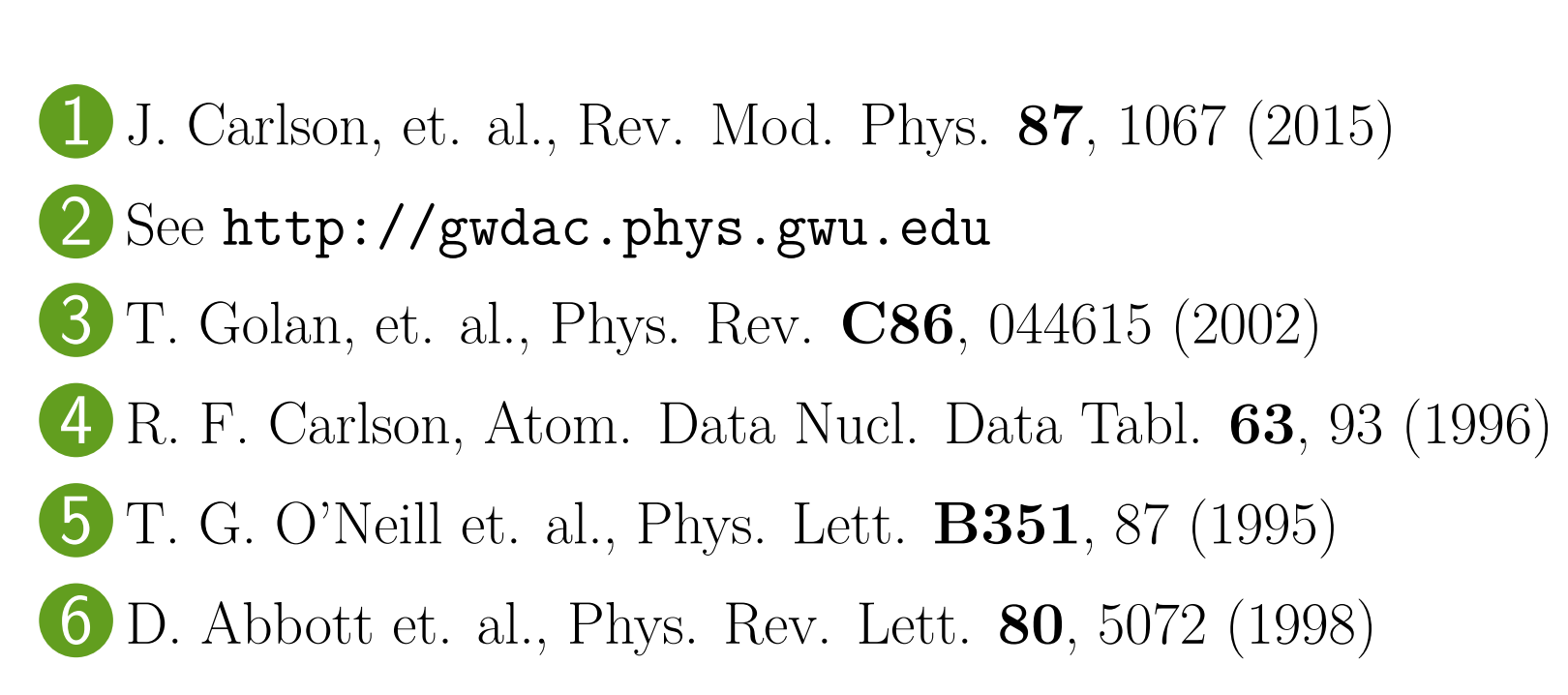

Contact Information 